\title{
The impact of lockdown and other stressors during the COVID-19 pandemic on depression and anxiety in a Lebanese opportunistic sample: an online cross-sectional survey
}

\author{
Rami Bou Khalil ${ }^{1,2}$ (D) Ramez Dagher ${ }^{1,2} \cdot$ Myriam Zarzour $^{1,2} \cdot$ Ghassan Sleilaty $^{2,3} \cdot$ Hanna Abi Akl $^{4}$ Maya Kallab² $^{2}$ \\ Sami Richa ${ }^{1,2}$
}

Accepted: 19 December 2021

๑) The Author(s), under exclusive licence to Springer Science+Business Media, LLC, part of Springer Nature 2022

\begin{abstract}
Lockdown measures were taken since February 2020 in Lebanon, a country already going through a socio-economic crisis, to fight the new coronavirus pandemic. The aim of this study is to evaluate the psychological impact of the lockdown in Lebanon. A cross-sectional, online survey was conducted during the lockdown period in order to punctually assess depression, anxiety symptoms as well as eating and substance use disorders using self-rating scales (the DASS-21, SCOFF and CAGE-AID respectively), while identifying factors that might affect those outcomes. Overall, 1133 participants completed the questionnaire. The DASS-21 score was positively correlated with the impact of lockdown on participants' lifestyle and lockdown duration. A highest effect on DASS-21 score was related to the intensity of other stressful life events that have happened during the last 3 months. Linear regression analysis also showed that age, monthly income, professional status, the SCOFF and CAGE-AID scores, the intensity of the lockdown's impact on lifestyle and having been through other stressful life events unrelated to the lockdown per se, were risk factors affecting significantly the DASS-21 score. Lockdown, as well as other stressful life event that have happened during the last 3 months, were therefore associated with higher depression, anxiety, and stress symptoms. These findings may guide future policy making strategies in order to prevent mental health problems in case of a pandemic concomitant with other critical stressors.
\end{abstract}

Keywords COVID-19 pandemic $\cdot$ Lockdown $\cdot$ Anxiety $\cdot$ Depression $\cdot$ Lebanon

\section{Introduction}

On the $11^{\text {th }}$ of March 2020, the World Health Organization described the Coronavirus-19 disease (COVID-19) as a pandemic after a virus that transmits through droplets

Rami Bou Khalil and Ramez Dagher ${ }^{\mathrm{a}}$ contributed equally to this work.

Rami Bou Khalil

rami.boukhalil@usj.edu.lb

1 Department of Psychiatry, Hôtel-Dieu de France Hospital, Beirut, Lebanon

2 Faculty of Medicine, Saint Joseph University, A. Naccache Boulevard Achrafieh- Beiruth, P.O. box: 166830, Lebanon, Lebanon

3 Clinical Research Centre, Hôtel-Dieu de France, Beirut, Lebanon

4 Yseop Lab, Paris, France and causes pneumonia, spread on international scale (Jin et al. 2020). Subsequently, multiple cities and countries around the world went into lockdown to contain the spread of the COVID-19 outbreak.

Avoidance behaviors as well as affective symptoms impacting daily routines of the community and the local economy have already been described during previous pandemics (Lau et al. 2010; Taylor et al. 2008; Ko et al. 2006), and the impact of the COVID-19 pandemic on lifestyle and wellbeing has likewise been of major interest in several affected populations (Dong and Bouey 2020; Liu et al. 2020; Zandifar and Badrfam 2020).

The psychological effects of isolation have already been described in the literature (Leigh-Hunt et al. 2017; Mollica 1993), but the magnitude of the lockdown that had been implemented worldwide during the COVID-19 pandemic remains unmatched (Mengin et al. 2020). In fact, the prevalence of many psychiatric illnesses has been noted to increase during times of isolation and lockdown, as 
psychological stressors tend to be more important (Mengin et al. 2020), and might even influence the likelihood to take precautionary anti-viral measures by the affected population (Leung 2003). Emergence of symptoms related to depressive disorders (Mengin et al. 2020; Torales et al. 2020) and post-traumatic stress disorder (Mengin et al. 2020; Torales et al. 2020; Bo et al. 2020; Sprang and Silman 2013) has been described in pandemics, as anxiety symptoms were found to appear during and after lockdown (Mengin, et al. 2020; Torales et al. 2020), and recent studies on COVID19 pandemic have also shown that quarantine could have a wide-ranging and a long-lasting effect on the quarantined population (Brooks et al. 2020).

A study that included 1210 respondents from 194 cities in China found that during the initial phase of the COVID-19 outbreak, more than half of the respondents rated the psychological impact of the pandemic as moderate-to-severe, while one-third of the respondents reported moderate-tosevere anxiety (Wang et al. 2020a, 2020b), and another study among 7143 students found that economic effects as well as delays in academic activities increased anxiety (Cao et al. 2020). Furthermore, healthcare workers who were at the frontline of the COVID-19 pandemic response were found to be particularly affected by psychological difficulties (Lai et al. 2020).

The literature also suggests a risk of worsening of preexisting eating disorders as well as an increased risk of developing de novo eating disorders, as lockdown makes food more accessible and available (Mengin et al. 2020). Research also described that during the lockdown, persons with substance use disorder had a tendency to increase their consumption of psychoactive substances, a frequent remedy for boredom, isolation, and anxiety whereas for others, the lockdown represented a period of great difficulty, as they found themselves deprived of drugs which might have led them to forced withdrawal (Mengin et al. 2020).

In Lebanon, where the prevalence of mood and anxiety disorders was estimated to be well above the $10 \%$ threshold before the pandemic (Karam et al. 2008), the first case of COVID-19 was reported on the 21st of February. Quick measures were taken to fight the pandemic: On the 28th of February, as only the 4th case of COVID-19 was being announced, the ministry of education closed schools and universities (Sly 2020). A state of general mobilization that imposed lockdown and called for lockdown for the general population was eventually announced by the Lebanese Council of Ministers and implemented on the 15th of March, with the government stopping all incoming flights to the country by the 18th of March (Sly 2020). Lockdown measures meant that all schools and universities were closed, and most public and private institutions and shops had to close their doors, as only supermarkets and healthcare establishments were allowed to remain open. This crisis has happened in a country already suffering from political turmoil and an uprising that had been ongoing since 2019 due to economic recession and unemployment (Hubbard 2020; Mounzer 2020).

We hypothesized that the impact of the lockdown during this COVID-19 pandemic in Lebanon is influenced by demographic, social, cultural, economic, legal and public health aspects in a way that renders the Lebanese individuals vulnerable to mental health problems such as depression, anxiety, eating and substance use disorders in a different manner than described elsewhere. Accordingly, the aim of the current study was to punctually evaluate, in an online survey underwent during the peak of lockdown, the direct psychological impact of this lockdown in the context of the COVID-19 pandemic in Lebanon. A secondary objective was to compare the impact of factors affecting depression, anxiety and stress in this context.

\section{Methods}

\section{Participants}

Participants needed to be more than 18 years old, living in Lebanon and master one of the following three languages used in Lebanon: French, English, or Arabic. This was an opportunistic sample of the Lebanese population. Overall, $\mathrm{N}=1133$ participants completed the study questionnaire. Participant's mean age was $29.2 \pm 9.6$ with female gender being predominant $(\mathrm{N}=812 ; 71.7 \%)$.

\section{Evaluation Tools}

The evaluation questionnaires consisted of:

1. A questionnaire regarding socio-demographic data (age, sex, nationality, region, monthly income, education, professional status, number of people at home, relationship status).

2. A question assessing the presence of a history of a known mental illness, taking psychotropic drugs, or a need for current or past psychological monitoring.

3. Two questions assessing the presence of a stressful event experienced during the last 3 months regardless of lockdown. In case a stressful event has been recently experienced by the participant, he/she will have to quantify the intensity of the stress experienced on a Likert-type scale ranging from 1 to 4 :

- Mild impact (It took me several days to adapt to the new situation).

- Moderate impact (the new situation affected me in a bearable way for a few weeks). 
- Severe impact (the new situation affected me in an unbearable way for several weeks).

- Very severe impact (I am still under the effect of the event experienced with unbearable physical and psychological symptoms).

4. Three questions assessing the lockdown related stressors as well as the impact and duration of the lockdown on the lifestyle in general), also allowing us to obtain four levels of impact:

- Level A: Absent impact (I continue to lead my life as usual without modifications due to the lockdown or with modifications due to the lockdown that I experience in a pleasant or normal way).

- Level B: Mild impact (I continue to lead my life as usual with the modifications due to the lockdown that I experience in a slightly unpleasant way)

- Level C: Moderate impact (My lifestyle has changed significantly with the modifications due to the lockdown I live in an unpleasant way but that I can bear).

- Level D: Severe impact (My lifestyle has changed drastically with the modifications due to the lockdown that I live in an unpleasant way and that I cannot bear)

5. The DASS questionnaire: The 21-item Depression Anxiety and Stress Scale questionnaire (DASS-21) in 3 versions (English, French and Arabic) to measure the importance of anxiety-depressive symptoms (Lovibond et al. 1995). The DASS-21 is made up of three parts of 7 specific questions related to depression, stress, and anxiety. Every sub-questionnaire has its own score that can be calculated for specific screening and a score for the DASS-21 as whole can be done for a further global assessment. The cut-off for moderate to severe depression is $\geq 7$ with a Cronbach's $\alpha=0.88$ in our sample. The cut-off for moderate to severe anxiety is $\geq 6$ with a Cronbach's $\alpha=0.86$ in our sample. The cut-off for moderate to severe stress is $\geq 10$ with a Cronbach's $\alpha=0.89$ in our sample. The DASS-21 was found to be a reliable and suitable for use to assess symptoms of common mental health problems, especially depression and anxiety among many populations (Le et al. 2017; Jiang et al. 2020). The DASS-21 total score had a Cronbach's $\alpha=0.93$.

6. The SCOFF questionnaire: The SCOFF questionnaire (Sick, Control, One stone, Fat, Food) with 5 items in 3 versions (English, French and Arabic) in order to measure the importance of vulnerability to eating disorders (Morgan et al. 1999). Answering by "yes" to two or more of the five questions has been related to a high probability of having an eating disorder. The SCOFF had a Cronbach's $\alpha=0.5$. Another question was added to assess perceived impact of the lockdown on eating disorders for those who had had a positive screening test.

7. The CAGE-AID questionnaire: The CAGE-AID questionnaire (Cut down, Annoyed, Guilty, Eye opener) with 4 items and in 3 versions (English, French and Arabic) to assess the difficulties with the consumption of alcohol and psychoactive substances (Brown and Rounds 1995). Answering by "yes" to two or more of the four questions has been related to a high probability of substance use disorder. The CAGE-AID had a Cronbach's $\alpha=0.71$. Another question was added to assess perceived impact of the lockdown on the consumption of alcohol and psychoactive substances for those who had had a positive screening test.

\section{Study Protocol}

An online survey (using Google Forms) through the most popular social communication networks (Facebook, Twitter, Linkedin and WhatsApp) was conducted between the $17^{\text {th }}$ of April and the $18^{\text {th }}$ of May 2020. This screening tool consisted of 47 questions that required approximately 15 min of the participant's time. Participants were asked for consent at the beginning at study, and upon completion, were sent an optional mail including their scores explaining the risk of having a clinically significant disorder. The study protocol has been approved by the ethics committee of Hotel Dieu de France hospital (file number CEHDF1632). The research protocol has been registered in the Lebanon Clinical Trials Registry (Primary registry indentifying number: LBCTR2020043459).

\section{Statistical Analysis}

All statistical analyses were conducted using JASP 0.12.2. Normality assumptions were assessed with Shapiro-Wilk test, and inspected visually using the Quartile-Quartile plots. Expectdly, the DASS-21 score was right-skewed. The comparison of the DASS-21 score with categorical variables was conducted using the Mann-Whitney U test and the KruskallWallis tests with stepdown post-hoc tests, as appropriate. The non parametric Spearman's rho coefficient was used for all the correlations. In the multiple linear regression analysis, the square root of DASS-21 total score was the dependent variable (having its distribution closer to normality assumptions) and all the other variables were entered as independent variables. A backward elimination of correlated variables and those with a high $\mathrm{p}$ value has been done while controlling the adjusted $\mathrm{R}^{2}$. The backward elimination of variables has been stopped when all independent variables became statistically significant. 


\section{Data Sharing Statement}

The current article includes the complete raw data-set collected in the study including the participants' data set, syntax file and $\log$ files for analysis. Pending acceptance for publication, all of the data files will be automatically uploaded to the Figshare repository.

\section{Results}

\section{Sample Characteristics}

Of all participants $(\mathrm{N}=1133)$ who completed the study questionnaire, 713 (62.9\%) lived in Mount Lebanon district (the closest district to the capital of Lebanon) followed by Beirut district, the capital of Lebanon $(\mathrm{N}=264 ; 23.3 \%)$. Most frequently, participants had an income higher than the equivalent of $3500 \$$ per month $(\mathrm{N}=331 ; 29.2 \%)$. Most participants had either a bachelor $(\mathrm{N}=364 ; 32.1 \%)$ or a master $(\mathrm{N}=472 ; 41.7 \%)$ university degree. In addition, most participants were either students $(\mathrm{N}=368 ; 32.5 \%)$ or employees $(\mathrm{N}=399 ; 35.2 \%)$. Socio-demographic characteristics are presented in Table 1.

Participants declared that the impact of the lockdown on their lifestyle was most frequently moderate $(\mathrm{N}=468$; 41.3\%). Assessed variables in relation to lockdown per se are presented in Table 2.

Finally, most participants $(\mathrm{N}=771 ; 68.0 \%)$ had been through a stressful life event in the 3 months period preceding the completion of the survey. Assessed variables in relation to this other stressful event are presented in Table 3. The sample's median scores and Q1-Q3 intervals on the different scales were: 36 (20-56) on the DASS-21 [divided into 14 (6-22), 6 (2-14), and 14 (6-22) on the depression, anxiety and stress subscales, respectively], 2 (1-3) on the SCOFF and $0(0-0)$ on the CAGE-AID.

\section{Factors Affecting DASS-21 Score}

In the correlation analysis with other continuous variables, DASS-21 score positively correlated with the level of lockdown impact on participants' life style having a moderate effect size (Spearman's rho $=0.424 ; \mathrm{p}<0.001$ ); lockdown duration having a small effect size (Spearman's rho $=0.077$; $\mathrm{p}=0.01$ ); the impact of other stressful life events on mental health having a large effect size (Spearman's rho $=0.506$; $\mathrm{p}<0.001$ ); SCOFF score with a large effect size (Spearman's rho $=0.55 ; \mathrm{p}<0.001)$; CAGE-AID score with a small effect size (Spearman's rho $=0.25 ; \mathrm{p}<0.001)$. Furthermore, DASS-21 score negatively correlated, with small effect sizes, with age (Spearman's rho $=-0.164 ; \mathrm{p}<0.001$ ); household monthly income (Spearman's rho $=-0.143 ; \mathrm{p}<0.001$ ); education level (Spearman's rho $=-0.164 ; \mathrm{p}<0.001$ ) (Table 4; Fig. 1).

Dichotomous variables that were found to be significantly affecting DASS-21 score were: gender (DASS median score for men $=32.0(14-48)$ vs. women $=38.0(20-58)$; $\mathrm{p}<0.001$ ); having a history of mental illness (DASS median score $=56.0(42-74)$ vs. $32.0(18-52)$ for those with no mental illness; $\mathrm{p}<0.001)$; having been through another stressful life event (DASS median score $=42.0$ (24-62) vs. 25.0 (12-41.5) for those not having been through stressful life events; $p<0.001$ ); having financial difficulties (DASS median score $=48.0(30-66)$ vs. $32.0(18-52)$ for those who have not; $\mathrm{p}<0.001$ ); having marital disputes (DASS median score $=62.0(44-84)$ vs. $34.0(18-54)$ for those who have not; $\mathrm{p}<0.001$ ); having been through a personal illness or an illness affecting a loved person (DASS median score $=47.0$ (28-68) vs. $34.0(18-54)$ for those who have not; $p<0.001)$; having experienced COVID-19 symptoms (DASS median score $=46.0(28-68)$ vs. $36.0(20-56)$ for those who have not; $p=0.02 \mathrm{~s} 8$ ); having worked in healthcare or any other public place (DASS median score $=32.0(18-52)$ vs. 36.0 (20-58) for those who have not; $\mathrm{p}=0.017$ ); having been in lockdown with a person with a physical illness (DASS median score $=40.0(31-63)$ vs. $34.0(18-56)$ for those who have not; $p<0.001)$. Other dichotomous variables which have been tested for a possible influence on DASS-21 are presented in Tables 1, 2 and 3.

DASS-21 score differed significantly according to the professional status (Kruskall-Wallis; $p<0.001$ ) with the post-hoc analysis showing that unemployed participants [DASS median score $=42(26-66)$ ], housewives [DASS median score $=38(28-68)$ ] and students [DASS median score $=41(24-62)]$ had a higher score than employers/ directors/managers [DASS median score $=26(12-36)$ ] $(p=0.002 ; p=0.01$ and $p<0.001$ respectively $)$ and participants exerting a liberal profession necessitating a diploma [DASS median score $=29(17.5-44)](p=0.003 ; p=0.033$ and $\mathrm{p}<0.001$ respectively). Moreover, DASS-21 score differed significantly according to the relationship status (Kruskall-Wallis; $\mathrm{p}=0.013$ ) with the post-hoc analysis showing that participants who were in a relationship [DASS median score $=40(22-58)]$ scored higher than married ones [DASS median score $=30(16-48)](p=0.013)$. No difference was found in DASS-21 score according to the region of residency (Kruskall-Wallis; $p=0.367$ ).

DASS-21 subscales for depression and anxiety were significantly different for the same socio-demographic parameters for which the DASS-21 total score has been found to be different (Table 1). In addition, DASS-21 subscales for depression and anxiety were significantly different for the same stressors related to COVID-19 pandemic and lockdown modalities for which the DASS-21 total score has been found to be different (Table 2). However, participants 
Table 1 DASS-21 scores according to socio-demographic characteristics of participants

\begin{tabular}{|c|c|c|c|c|c|c|c|c|c|}
\hline Variable & Category & $\begin{array}{l}\text { Frequency } \\
(\%)\end{array}$ & $\begin{array}{l}\text { DASS-21 } \\
\text { score median } \\
\text { (Q1-Q3) }\end{array}$ & Test & $\mathrm{P}$ value & $\begin{array}{l}\text { Depression } \\
\text { score subscale } \\
\text { median (Q1- } \\
\text { Q3) }\end{array}$ & $\begin{array}{l}\text { Test and } \mathrm{P} \\
\text { value }\end{array}$ & $\begin{array}{l}\text { Anxiety score } \\
\text { subscale } \\
\text { median (Q1- } \\
\text { Q3) }\end{array}$ & $\begin{array}{l}\text { Test and } \mathrm{P} \\
\text { value }\end{array}$ \\
\hline \multirow[t]{2}{*}{ Gender } & Male & $321(28.3 \%)$ & $32.0(14-48)$ & $\mathrm{MWU}^{\mathrm{c}}$ & $<0.001$ & $6.7(3-10)$ & MWU & $3.6(1-5)$ & \multirow{2}{*}{$\begin{array}{l}\text { MWU } \\
<\mathbf{0 . 0 0 1}\end{array}$} \\
\hline & Female & $812(71.7 \%)$ & $38.0(20-58)$ & & & $7.8(4-11)$ & $<0.001$ & $5.04(2-7)$ & \\
\hline \multirow[t]{2}{*}{ Nationality } & Lebanese & $1110(97.9 \%)$ & $36.0(20-56)$ & MWU & 0.577 & $7.5(3-11)$ & MWU & $4.6(1-7)$ & MWU \\
\hline & Non-Lebanese & $23(2.1 \%)$ & $36.0(19-69)$ & & & $8.3(3-12)$ & \multirow[t]{2}{*}{0.521} & $5.4(1-9)$ & \multirow[t]{2}{*}{0.558} \\
\hline \multirow[t]{3}{*}{ Residency } & Beirut & $264(23.3 \%)$ & $36.0(26-54)$ & \multirow[t]{3}{*}{$\mathrm{KW}^{\mathrm{c}}$} & \multirow[t]{3}{*}{0.367} & $7.4(4-11)$ & & $4.5(1-7)$ & \\
\hline & $\begin{array}{l}\text { Mount Leba- } \\
\text { non }\end{array}$ & $760(67.1 \%)$ & $36.0(20-58)$ & & & $7.6(3-11)$ & KW & $4.7(1-7)$ & KW \\
\hline & Other & $109(9.6 \%)$ & $38.0(18-54)$ & & & $7.1(3-11)$ & 0.678 & $4.3(1-7)$ & 0.936 \\
\hline \multirow{8}{*}{$\begin{array}{l}\text { Household } \\
\text { monthly } \\
\text { income }\end{array}$} & $\begin{array}{l}\text { Less than } \\
500 \$\end{array}$ & $50(4.4 \%)$ & $\begin{array}{l}41.0 \\
\quad(28.5-64)\end{array}$ & \multirow[t]{8}{*}{ KW } & \multirow[t]{8}{*}{$<0.001$} & $9.02(6-12)$ & \multirow[t]{8}{*}{$\begin{array}{l}\text { KW } \\
<\mathbf{0 . 0 0 1}\end{array}$} & $5.4(2-9)$ & \multirow[t]{8}{*}{$\begin{array}{l}\text { KW } \\
<\mathbf{0 . 0 0 1}\end{array}$} \\
\hline & $500-1000 \$$ & $120(10.6 \%)$ & $\begin{array}{l}38.0 \\
\quad(24-56.5)\end{array}$ & & & $8.3(4-11)$ & & $4.8(1.7-7)$ & \\
\hline & $1000-1500 \$$ & $191(16.9 \%)$ & $46.0(24-68)$ & & & $8.5(4-12)$ & & $6.7(2-9.5)$ & \\
\hline & $1500-2000 \$$ & $143(12.6 \%)$ & $38.0(21-58)$ & & & $8.2(4-12)$ & & $4.7(2-6)$ & \\
\hline & $2000-2500 \$$ & $103(9.1 \%)$ & $32.0(16-49)$ & & & $6.8(3-9.5)$ & & $4.01(1-6)$ & \\
\hline & $2500-3000 \$$ & $90(7.9 \%)$ & $44.0(23-58)$ & & & $7.9(4-11.7)$ & & $4.5(2-7.7)$ & \\
\hline & $3000-3500 \$$ & $105(9.3 \%)$ & $30.0(16-50)$ & & & $6.7(3-10)$ & & $3.8(1-5)$ & \\
\hline & $\begin{array}{l}\text { More than } \\
3500 \$\end{array}$ & $331(29.2 \%)$ & $30.0(18-50)$ & & & $6.6(3-9)$ & & $4.01(1-6)$ & \\
\hline \multirow[t]{6}{*}{$\begin{array}{c}\text { Education } \\
\text { level }\end{array}$} & No diploma & $8(0.7 \%)$ & $\begin{array}{l}55.0(43.5- \\
60.5)\end{array}$ & $\mathrm{KW}$ & $<0.001$ & $\begin{array}{l}10.1 \\
(7.7-10.7)\end{array}$ & \multirow[t]{6}{*}{$\begin{array}{l}\text { KW } \\
<\mathbf{0 . 0 0 1}\end{array}$} & $6.6(5-9.2)$ & \multirow[t]{6}{*}{$\begin{array}{l}\text { KW } \\
<\mathbf{0 . 0 0 1}\end{array}$} \\
\hline & $\begin{array}{l}\text { Middle school } \\
\text { diploma }\end{array}$ & $14(1.2 \%)$ & $\begin{array}{l}30.0 \\
\quad(12-102.5)\end{array}$ & & & $8.4(2-17)$ & & $7.6(0-17.5)$ & \\
\hline & $\begin{array}{l}\text { High school } \\
\text { diploma }\end{array}$ & $142(12.5 \%)$ & $43.0(26-68)$ & & & $9(5-13)$ & & $6.2(2-10)$ & \\
\hline & Bachelor & $364(32.1 \%)$ & $40.0(22-60)$ & & & $8.1(4-12)$ & & $4.9(2-8)$ & \\
\hline & Masters & $472(41.7 \%)$ & $34.0(18-50)$ & & & $6.8(3-10)$ & & $4.1(1-6)$ & \\
\hline & Doctorate & $133(11.7 \%)$ & $30.0(14-48)$ & & & $6.6(3-9)$ & & $3.5(1-6)$ & \\
\hline \multirow{7}{*}{$\begin{array}{l}\text { Number of } \\
\text { individuals } \\
\text { in house- } \\
\text { hold }\end{array}$} & 1 & $49(4.3 \%)$ & $40.0(18-50)$ & KW & 0.748 & $7.6(4-11)$ & \multirow{7}{*}{$\begin{array}{l}\text { KW } \\
0.405\end{array}$} & $4.3(1-7)$ & \multirow{7}{*}{$\begin{array}{l}\text { KW } \\
0.808\end{array}$} \\
\hline & 2 & $210(18.5 \%)$ & $\begin{array}{l}34.0(16.5- \\
53.5)\end{array}$ & & & $7.05(3-10)$ & & $4.4(1-6)$ & \\
\hline & 3 & $268(23.6 \%)$ & $38.0(18-58)$ & & & $7.9(4-11)$ & & $4.8(1-7.2)$ & \\
\hline & 4 & $296(26.1 \%)$ & $34.0(20-56)$ & & & $7.5(3.7-11)$ & & $4.6(1-7)$ & \\
\hline & 5 & $176(15.5 \%)$ & $33.0(18-56)$ & & & $7.4(3-11)$ & & $4.4(1-7)$ & \\
\hline & 6 & $92(8.1 \%)$ & $\begin{array}{l}37.0 \\
\quad(23.5-59)\end{array}$ & & & $7.4(4-11)$ & & $4.8(1.7-7)$ & \\
\hline & 7 & $42(3.7 \%)$ & $38.0(30-56)$ & & & $8.8(6-13)$ & & $5.1(2-7)$ & \\
\hline \multirow{6}{*}{$\begin{array}{l}\text { Current } \\
\text { relational } \\
\text { status }\end{array}$} & Single & $559(49.3 \%)$ & $38.0(20-58)$ & \multirow[t]{6}{*}{ KW } & 0.013 & $8.1(4-11)$ & \multirow{6}{*}{$\begin{array}{l}\text { KW } \\
<\mathbf{0 . 0 0 1}\end{array}$} & $4.7(1.5-7)$ & \multirow{6}{*}{$\begin{array}{l}\text { KW } \\
<\mathbf{0 . 0 0 1}\end{array}$} \\
\hline & $\begin{array}{l}\text { In a relation- } \\
\text { ship }\end{array}$ & $259(22.9 \%)$ & $40.0(22-58)$ & & & $7.7(3-11.5)$ & & $5.3(1-8)$ & \\
\hline & Engaged & $41(3.6 \%)$ & $36.0(18-52)$ & & & $7.1(4-9)$ & & $4.4(2-8)$ & \\
\hline & Married & $252(22.3 \%)$ & $30.0(16-48)$ & & & $6.3(2.7-9)$ & & $3.8(1-5)$ & \\
\hline & Separated & $8(0.7 \%)$ & $\begin{array}{l}49.0 \\
\quad(25.5-64)\end{array}$ & & & $8.2(2.7-13.2)$ & & $3.3(0.7-4.7)$ & \\
\hline & Divorced & $14(1.2 \%)$ & $24.0(12-44)$ & & & $6.5(3-9.2)$ & & $2.2(1-3.7)$ & \\
\hline
\end{tabular}


Table 1 (continued)

\begin{tabular}{|c|c|c|c|c|c|c|c|c|c|}
\hline Variable & Category & $\begin{array}{l}\text { Frequency } \\
(\%)\end{array}$ & $\begin{array}{l}\text { DASS-21 } \\
\text { score median } \\
\text { (Q1-Q3) }\end{array}$ & Test & $\mathrm{P}$ value & $\begin{array}{l}\text { Depression } \\
\text { score subscale } \\
\text { median (Q1- } \\
\text { Q3) }\end{array}$ & $\begin{array}{l}\text { Test and } \mathrm{P} \\
\text { value }\end{array}$ & $\begin{array}{l}\text { Anxiety score } \\
\text { subscale } \\
\text { median (Q1- } \\
\text { Q3) }\end{array}$ & $\begin{array}{l}\text { Test and } P \\
\text { value }\end{array}$ \\
\hline \multirow{8}{*}{$\begin{array}{l}\text { Current } \\
\text { professional } \\
\text { status }\end{array}$} & Unemployed & $81(7.2 \%)$ & $42.0(26-66)$ & \multirow[t]{8}{*}{ KW } & \multirow[t]{8}{*}{$<0.001$} & $9.1(5-13)$ & \multirow{8}{*}{$\begin{array}{l}\text { KW } \\
<\mathbf{0 . 0 0 1}\end{array}$} & $5.4(2-8)$ & \multirow{8}{*}{$\begin{array}{l}\text { KW } \\
<\mathbf{0 . 0 0 1}\end{array}$} \\
\hline & Housewife & $41(3.6 \%)$ & $38.0(28-68)$ & & & $8.8(3-13)$ & & $5.3(2-7)$ & \\
\hline & Student & $368(32.5 \%)$ & $41.0(24-62)$ & & & $8.5(4-12)$ & & $5.3(2-8)$ & \\
\hline & Retired & $7(0.6 \%)$ & $14.0(8-39)$ & & & $6.2(2-7)$ & & $3.5(1-4.5)$ & \\
\hline & $\begin{array}{l}\text { Liberal } \\
\text { profession }^{\mathrm{a}}\end{array}$ & $21(1.9 \%)$ & $38.0(22-56)$ & & & $6.9(4-10)$ & & $4.2(2-7)$ & \\
\hline & Employee & $399(35.2 \%)$ & $36.0(20-52)$ & & & $7.1(3-10)$ & & $4.5(1-7)$ & \\
\hline & $\begin{array}{l}\text { Employer/ } \\
\text { Director/ } \\
\text { Manager }\end{array}$ & $56(4.9 \%)$ & $\begin{array}{l}26.0 \\
\quad(12-36.5)\end{array}$ & & & $5.1(2-7)$ & & $2.9(1-4.2)$ & \\
\hline & $\begin{array}{l}\text { Liberal } \\
\text { profession }^{\mathrm{b}}\end{array}$ & $160(14.1 \%)$ & $\begin{array}{l}29.0 \\
(17.5-44)\end{array}$ & & & $6.01(3-8)$ & & $3.3(1-5)$ & \\
\hline \multirow{2}{*}{$\begin{array}{l}\text { Personal } \\
\text { history of } \\
\text { mental ill- } \\
\text { ness }\end{array}$} & Yes & $157(13.9 \%)$ & $56.0(42-74)$ & \multirow[t]{2}{*}{ MWU } & \multirow[t]{2}{*}{$<0.001$} & $10.4(7-14)$ & \multirow{2}{*}{$\begin{array}{l}\text { MWU } \\
<\mathbf{0 . 0 0 1}\end{array}$} & $7.7(4-11)$ & \multirow{2}{*}{$\begin{array}{l}\text { MWU } \\
<\mathbf{0 . 0 0 1}\end{array}$} \\
\hline & No & $976(86.1 \%)$ & $32.0(18-52)$ & & & $7.1(3-10)$ & & $4.1(1-6)$ & \\
\hline
\end{tabular}

a: Not requiring specialization diploma

b: Requiring specialization diploma (doctor, engineer, lawyer, etc.)

c: MWU = Mann-Whitney-U test; KW = Kruskal-Wallis test

$p$-values numbers marked in bold indicate numbers that are significant on the $95 \%$ confidence limit

who personally experienced COVID-19 symptoms presented significantly different DASS-21 scores for anxiety but not for depression. Moreover, participants who reported wearing masks and gloves in public places presented a higher anxiety subscale score without any difference on the DASS-21 total score or the depression subscale. DASS-21 subscales for depression and anxiety were significantly different for stressors unrelated to lockdown for which the DASS-21 total score has been found to be different (Table 3). However, loss of work and separation have been found to be affecting the DASS-21 score with a higher depression rather than a higher anxiety while having experienced a traffic accident was with higher anxiety than depression.

\section{Regression Model}

Statistically significant factors affecting $40.3 \%$ of the variability (Model's adjusted R square $=0.403$ ) of the square root transform of DASS-21 were: age $(\mathrm{p}<0.001)$; monthly income $(p=0.031)$; education level $(p<0.001)$; the intensity of current lockdown impact on life style $(\mathrm{p}<0.001)$; the intensity of other stressful life event during the last 3 months $(\mathrm{p}<0.001)$ and CAGE-AID score $(\mathrm{p}<0.001)$. Of note, the highest variability effect on DASS-21 score was related to the intensity of other stressful life event which happened during the last 3 months (Standardized coefficient $=0.382$ ) followed by the intensity of current lockdown impact on life style $($ Standardized coefficient $=0.308)($ Table 5$)$.

\section{Discussion}

To our knowledge, this is the first study that assesses the impact of lockdown, per se as well as the global impact of the pandemic, on the mental health of the general population in Lebanon. During last three months as compared to before, the impact of the lockdown was found to be correlated to an increased severity of the depression, anxiety and stress symptoms. This is congruent to data from the literature in which isolation was found to have a significant impact on mental health (Leigh-Hunt et al. 2017; Mollica 1993). As a matter of fact, recent data coming from compiled studies investigating mental health of individuals living through the pandemic show that health care-workers manifest increased depression, anxiety, psychological distress and poor sleep quality. As for the general public, available studies point towards lower psychological well-being and higher scores of anxiety and depression when compared to the ordinary state with no increase in the levels of these symptoms after a few weeks of lockdown (Vindegaard and Benros 2020). It 
Table 2 DASS-21 scores according to variables related to the pandemic and the lockdown

\begin{tabular}{|c|c|c|c|c|c|c|c|c|c|}
\hline Variable & Category & $\begin{array}{l}\text { Frequency } \\
(\%)\end{array}$ & $\begin{array}{l}\text { DASS-21 } \\
\text { score median } \\
\text { (Q1-Q3) }\end{array}$ & Test & $\mathrm{P}$ value & $\begin{array}{l}\text { DASS-21 } \\
\text { subscale score } \\
\text { for depression }\end{array}$ & $\begin{array}{l}\text { Test and } p \\
\text { value }\end{array}$ & $\begin{array}{l}\text { DASS-21 } \\
\text { subscale for } \\
\text { anxiety }\end{array}$ & $\begin{array}{l}\text { Test and } p \\
\text { value }\end{array}$ \\
\hline \multirow{4}{*}{$\begin{array}{l}\text { Level of } \\
\text { impact of } \\
\text { lockdown } \\
\text { on par- } \\
\text { ticipants' } \\
\text { lifestyle }\end{array}$} & Absent & $123(10.9 \%)$ & $14.0(5-31)$ & \multirow{4}{*}{$\begin{array}{l}\text { Spearman's } \\
\text { rho }=0.424\end{array}$} & \multirow[t]{4}{*}{$<0.001$} & $3.7(1-6)$ & \multirow{4}{*}{$\begin{array}{l}\text { Spearman's } \\
\text { rho }=0.436 \\
(<\mathbf{0 . 0 0 1})\end{array}$} & $2.6(0-4)$ & \multirow{4}{*}{$\begin{array}{r}\text { Spearman's } \\
\text { rho }=0.318 \\
(<\mathbf{0 . 0 0 1 )}\end{array}$} \\
\hline & Mild & $434(38.3 \%)$ & $28.0(14-48)$ & & & $6.3(2-9)$ & & $3.9(1-6)$ & \\
\hline & Moderate & $468(41.3 \%)$ & $40.0(26-58)$ & & & $8.4(5-11)$ & & $3.8(2-7)$ & \\
\hline & Severe & $108(9.5 \%)$ & $\begin{array}{l}68.0 \\
\quad(47.5-86)\end{array}$ & & & $13.2(9-18)$ & & $8.5(4-12)$ & \\
\hline \multirow{6}{*}{$\begin{array}{l}\text { Duration of } \\
\text { lockdown } \\
\text { in weeks }\end{array}$} & 1 & $45(3.9 \%)$ & $28.0(14-50)$ & \multirow{6}{*}{$\begin{array}{l}\text { Spearman's } \\
\text { rho }=0.077\end{array}$} & \multirow[t]{6}{*}{0.010} & $6.2(2-9)$ & \multirow{6}{*}{$\begin{array}{l}\text { Spearman's } \\
\text { rho }=0.089 \\
(\mathbf{0 . 0 0 3 )}\end{array}$} & $4.04(1-5)$ & \multirow{6}{*}{$\begin{array}{l}\text { Spearman's } \\
\text { rho }=0.078 \\
(\mathbf{0 . 0 0 9 )}\end{array}$} \\
\hline & 2 & $26(2.3 \%)$ & $\begin{array}{l}31.0 \\
\quad(22-55.5)\end{array}$ & & & $6.3(3-9)$ & & $4.3(1-6.5)$ & \\
\hline & 3 & $106(9.4 \%)$ & $30.0(18-54)$ & & & $7.1(3-10)$ & & $3.9(1-6)$ & \\
\hline & 4 & $420(37.1 \%)$ & $36.0(20-54)$ & & & $7.3(3-11)$ & & $4.5(1-6)$ & \\
\hline & 5 & $345(30.5 \%)$ & $34.0(20-56)$ & & & $7.6(4-11)$ & & $4.6(1-7)$ & \\
\hline & 6 & $191(16.8 \%)$ & $40.0(21-64)$ & & & $8.5(4-12)$ & & $5.6(1-9)$ & \\
\hline \multirow{2}{*}{$\begin{array}{l}\text { Returned } \\
\text { from a trip } \\
\text { abroad }\end{array}$} & Yes & $69(6.1 \%)$ & $36.0(20-56)$ & \multirow[t]{2}{*}{$\mathrm{MWU}^{\mathrm{a}}$} & \multirow[t]{2}{*}{0.105} & $7.5(3-11)$ & MWU & $4.6(1-7)$ & MWU \\
\hline & No & $1064(93.9 \%)$ & $44.0(22-62)$ & & & $8.4(4-12)$ & $(0.127)$ & $5.08(1-9)$ & $(0.403)$ \\
\hline \multirow{2}{*}{$\begin{array}{c}\text { Experienced } \\
\text { COVID-19 } \\
\text { symptoms }\end{array}$} & Yes & $49(4.3 \%)$ & $46.0(28-68)$ & \multirow[t]{2}{*}{ MWU } & \multirow[t]{2}{*}{0.028} & $8.3(4-13)$ & MWU & $6.4(3-9)$ & MWU \\
\hline & No & $1084(95.7 \%)$ & $36.0(20-56)$ & & & $7.5(3-11)$ & $(0.441)$ & $4.5(1-7)$ & $(0.007)$ \\
\hline \multirow[b]{2}{*}{$\begin{array}{l}\text { Contact with } \\
\text { people } \\
\text { diagnosed } \\
\text { or sus- } \\
\text { pected of } \\
\text { having the } \\
\text { virus }\end{array}$} & Yes & $88(7.8 \%)$ & $32.0(18-52)$ & \multirow[t]{2}{*}{ MWU } & \multirow[t]{2}{*}{0.333} & $7.3(3-10)$ & MWU & $4.4(1-7.2)$ & MWU \\
\hline & No & $1045(92.2 \%)$ & $36.0(20-56)$ & & & $7.5(3-11)$ & $(0.662)$ & $4.4(1-7)$ & $(0.211)$ \\
\hline \multirow{2}{*}{$\begin{array}{l}\text { Tested } \\
\text { positive for } \\
\text { COVID-19 }\end{array}$} & Yes & $7(0.7 \%)$ & $42.0(13-73)$ & \multirow[t]{2}{*}{ MWU } & \multirow[t]{2}{*}{0.872} & $10.2(2-17)$ & MWU & $5(0.5-7)$ & MWU \\
\hline & No & $1126(99.3 \%)$ & $36.0(20-56)$ & & & $7.5(3-11)$ & $(0.464)$ & $4.6(1-7)$ & $(0.401)$ \\
\hline \multirow{2}{*}{$\begin{array}{l}\text { Worn masks/ } \\
\text { gloves in } \\
\text { public } \\
\text { places most } \\
\text { of the time }\end{array}$} & Yes & $830(73.3 \%)$ & $36.0(20-56)$ & \multirow[t]{2}{*}{ MWU } & \multirow[t]{2}{*}{0.282} & $7.5(4-11)$ & MWU & $4.7(1.2-7)$ & MWU \\
\hline & No & $303(26.7 \%)$ & $36.0(16-55)$ & & & $7.5(3-11)$ & $(0.868)$ & $4.3(1-7)$ & $(0.045)$ \\
\hline \multirow{2}{*}{$\begin{array}{l}\text { Worked in } \\
\text { healthcare } \\
\text { or a public } \\
\text { place at } \\
\text { risk }\end{array}$} & Yes & $239(21.1 \%)$ & $32.0(18-52)$ & \multirow[t]{2}{*}{ MWU } & \multirow[t]{2}{*}{0.017} & $7(3-10)$ & MWU & $3.8(1-6)$ & MWU \\
\hline & No & $894(78.9 \%)$ & $36.0(20-58)$ & & & $7.7(4-11)$ & $(\mathbf{0 . 0 3 9})$ & $4.8(1-7)$ & $(\mathbf{0 . 0 0 3 )}$ \\
\hline \multirow[b]{2}{*}{$\begin{array}{l}\text { Being in } \\
\text { lockdown } \\
\text { with a } \\
\text { person with } \\
\text { significant } \\
\text { health } \\
\text { problems }\end{array}$} & Yes & $107(9.4 \%)$ & $40.0(31-63)$ & \multirow[t]{2}{*}{ MWU } & \multirow[t]{2}{*}{$<0.001$} & $8.6(5-11.5)$ & MWU & $5.6(2-8)$ & MWU \\
\hline & No & $1026(90.6 \%)$ & $34.0(18-56)$ & & & $7.4(3-11)$ & $(0.025)$ & $4.5(1-7)$ & $(0.006)$ \\
\hline Worked from & Yes & $623(55 \%)$ & $34.0(19-56)$ & MWU & 0.281 & $7.2(3-11)$ & MWU & $4.6(1-7)$ & MWU \\
\hline home & No & $510(45 \%)$ & $38.0(20-56)$ & & & $7.9(3.2-11)$ & $(0.061)$ & $4.6(1-7)$ & $(0.773)$ \\
\hline
\end{tabular}

a: MWU = Mann-Whitney-U test

$p$-values numbers marked in bold indicate numbers that are significant on the $95 \%$ confidence limit

has been hypothesized that these symptoms of depression and anxiety may become excessive whenever individuals with certain types of brain biobehavioural types such as those with a ruminative, anhedonic or "threat dysregulation" styles become exposed to pandemic-related cues (Hagerty and Williams 2020). Fortunately, symptoms of anxiety and 
Table 3 DASS-21 scores according to variables related to stressors unrelated to lockdown (in the previous 3 months before participating to the survey)

\begin{tabular}{|c|c|c|c|c|c|c|c|c|c|}
\hline Variable & Category & $\begin{array}{l}\text { Frequency } \\
(\%)\end{array}$ & $\begin{array}{l}\text { DASS-21 } \\
\text { score median } \\
\text { (Q1-Q3) }\end{array}$ & Test & $\mathrm{P}$ value & $\begin{array}{l}\text { DASS-21 } \\
\text { subscale } \\
\text { score for } \\
\text { depression }\end{array}$ & $\begin{array}{l}\text { Test and } p \\
\text { value }\end{array}$ & $\begin{array}{l}\text { DASS-21 } \\
\text { subscale } \\
\text { score for } \\
\text { anxiety }\end{array}$ & $\begin{array}{l}\text { Test and } p \\
\text { value }\end{array}$ \\
\hline \multirow{2}{*}{$\begin{array}{l}\text { Presence of } \\
\text { another } \\
\text { stressful } \\
\text { event dur- } \\
\text { ing the last } \\
\mathbf{3} \text { months }\end{array}$} & Yes & $771(68 \%)$ & $42.0(24-62)$ & $M_{W}{ }^{a}$ & $<0.001$ & $8.4(4-12)$ & MWU & $5.3(2-8)$ & MWU \\
\hline & No & $362(32 \%)$ & $\begin{array}{l}25.0 \\
\quad(12-41.5)\end{array}$ & & & $5.5(2-8)$ & $(<0.001)$ & $3.1(1-5)$ & $(<0.001)$ \\
\hline \multirow{2}{*}{$\begin{array}{l}\text { Difficulties } \\
\text { at work }\end{array}$} & Yes & $292(25.8 \%)$ & $42.0(24-58)$ & MWU & $<0.001$ & $8.2(5-11)$ & MWU & $5.6(2-7)$ & MWU \\
\hline & No & $841(74.2 \%)$ & $34.0(18-56)$ & & & $7.3(3-110$ & $(0.001)$ & $4.5(1-7)$ & $(0.005)$ \\
\hline \multirow[t]{2}{*}{ Loss of work } & Yes & $62(5.5 \%)$ & $\begin{array}{l}44.0 \\
\quad(34-65.5)\end{array}$ & MWU & 0.004 & $9.6(8-11.7)$ & MWU & $5.4(2-8)$ & MWU \\
\hline & No & $1071(94.5 \%)$ & $36.0(18-56)$ & & & $7.4(3-11)$ & $(<0.001)$ & $4.6(1-7)$ & $(0.124)$ \\
\hline \multirow{2}{*}{$\begin{array}{l}\text { Financial } \\
\text { difficulties }\end{array}$} & Yes & $253(22.3 \%)$ & $48.0(30-66)$ & MWU & $<0.001$ & $9.3(6-13)$ & MWU & $5.8(2-9)$ & MWU \\
\hline & No & $880(77.7 \%)$ & $32.0(18-52)$ & & & $7.06(3-10)$ & $(<0.001)$ & $4.3(1-6)$ & $(<0.001)$ \\
\hline \multirow[t]{2}{*}{ Legal issues } & Yes & $13(1.1 \%)$ & $46.0(18-64)$ & MWU & 0.518 & $8.3(2-11)$ & MWU & $5.07(2-8)$ & MWU \\
\hline & No & $1120(98.9 \%)$ & $36.0(20-56)$ & & & $7.5(3-11)$ & $(0.676)$ & $4.6(1-7)$ & $(0.529)$ \\
\hline \multirow{2}{*}{$\begin{array}{l}\text { Marital } \\
\text { disputes }\end{array}$} & Yes & $71(6.3 \%)$ & $62.0(44-84)$ & MWU & $<0.001$ & $11.3(8-15)$ & MWU & $7.6(3-11.5)$ & MWU \\
\hline & No & $1062(93.7 \%)$ & $34.0(18-54)$ & & & $7.3(3-11)$ & $(<0.001)$ & $4.4(1-7)$ & $(<0.001)$ \\
\hline \multirow[t]{2}{*}{ Separation } & Yes & $62(5.5 \%)$ & $46.0(26-68)$ & MWU & 0.008 & $9.3(5-13)$ & MWU & $5.7(1.2-8.7)$ & MWU \\
\hline & No & $1071(94.5 \%)$ & $36.0(20-56)$ & & & $7.4(3-11)$ & $(\mathbf{0 . 0 1})$ & $4.5(1-7)$ & $(0.118)$ \\
\hline \multirow[t]{2}{*}{ Divorce } & Yes & $3(0.3 \%)$ & $22.0(17-70)$ & MWU & 0.908 & $8.6(2.5-12)$ & MWU & $7(1-10.5)$ & MWU \\
\hline & No & $1130(99.7 \%)$ & $36.0(20-56)$ & & & $7.5(3-11)$ & $(0.789)$ & $4.6(1-7)$ & $(0.829)$ \\
\hline \multirow{2}{*}{$\begin{array}{l}\text { Been } \\
\text { through } \\
\text { a traffic } \\
\text { accident }\end{array}$} & Yes & $21(1.9 \%)$ & $56.0(38-62)$ & MWU & 0.003 & $9.2(7-11)$ & MWU & $7.3(4-9)$ & MWU \\
\hline & No & $1112(98.1 \%)$ & $36.0(20-56)$ & & & $7.5(3-11)$ & $(0.081)$ & $4.6(1-7)$ & $(<0.001)$ \\
\hline \multirow{2}{*}{$\begin{array}{l}\text { Personal } \\
\text { illness or } \\
\text { illness of a } \\
\text { loved one }\end{array}$} & Yes & $120(10.6 \%)$ & $47.0(28-68)$ & MWU & $<0.001$ & $8.9(5-12)$ & MWU & $6.3(2-9)$ & MWU \\
\hline & No & $1013(89.4 \%)$ & $34.0(18-54)$ & & & $7.4(3-11)$ & $(\mathbf{0 . 0 0 3 )}$ & $4.4(1-7)$ & $(<0.001)$ \\
\hline \multirow[t]{2}{*}{$\begin{array}{l}\text { Death of a } \\
\text { loved one }\end{array}$} & Yes & $60(5.3 \%)$ & $\begin{array}{l}43.0 \\
\quad(29.5-68)\end{array}$ & MWU & 0.006 & $9.1(4-14.2)$ & MWU & $6.6(2-9)$ & MWU \\
\hline & No & $1073(94.7 \%)$ & $36.0(20-56)$ & & & $7.4(3-11)$ & $(\mathbf{0 . 0 3 1})$ & $4.5(1-7)$ & $(0.003)$ \\
\hline \multirow{2}{*}{$\begin{array}{l}\text { Other stress- } \\
\text { ful events }\end{array}$} & Yes & $307(27.1 \%)$ & $46.0(30-64)$ & MWU & $<0.001$ & $9.2(6-13)$ & MWU & $5.8(2-9)$ & MWU \\
\hline & No & $826(72.9 \%)$ & $32.0(16-52)$ & & & $6.9(3-10)$ & $(<0.001)$ & $4.2(1-6)$ & $(<0.001)$ \\
\hline \multirow{5}{*}{$\begin{array}{l}\text { Intensity of } \\
\text { the stress- } \\
\text { ful life } \\
\text { event }\end{array}$} & No stressors & $369(32.6 \%)$ & - & Spearman's & $<0.001$ & $5.5(2-8)$ & Spearma & $3.1(1-5)$ & Spearma \\
\hline & Mild impact & $231(20.4 \%)$ & $30(12-42)$ & $\mathrm{rch}$ & & $5.5(2-8)$ & n's rho $=$ & $3.5(1-5)$ & \\
\hline & $\begin{array}{l}\text { Moderate } \\
\text { impact }\end{array}$ & $330(29.1 \%)$ & $40(26-56)$ & & & $8.2(5-11)$ & $\begin{array}{l}0.43 \\
(<\mathbf{0 . 0 0 1})\end{array}$ & $4.9(2-7)$ & $\begin{array}{l}0.402 \\
(<\mathbf{0 . 0 0 1})\end{array}$ \\
\hline & $\begin{array}{l}\text { Severe } \\
\text { impact }\end{array}$ & $156(12.8 \%)$ & $60(40-74)$ & & & $10.7(8-14)$ & & $7.5(3-11)$ & \\
\hline & $\begin{array}{l}\text { Very severe } \\
\text { impact }\end{array}$ & $47(4.1 \%)$ & $80(65-100)$ & & & $15.6(12-19)$ & & $10.5(6-13)$ & \\
\hline
\end{tabular}

a: $\mathrm{MWU}=$ Mann-Whitney-U test

$p$-values numbers marked in bold indicate numbers that are significant on the $95 \%$ confidence limit

depression seem to be reversible as soon as the threat related to the pandemic starts to diminish (Sønderskov et al. 2020).

In the context of the Lebanese population, other factors were found to be at least as impactful as the lockdown itself. Our study found that the anxiety, depressive and stress scores were even more correlated to other stressors, as the highest variability effect on DASS-21 score was related to the intensity of other stressful life event which happened 
Table 4 Continuous variables' correlation with the DASS-21 score

\begin{tabular}{llr}
\hline Variable & $\begin{array}{l}\text { Correlation coefficient with } \\
\text { DASS-21 score-Spear- } \\
\text { man's Rho }\end{array}$ & P value \\
& -0.164 & $<0.001$ \\
Age & -0.143 & $<0.001$ \\
Monthly income & -0.164 & $<0.001$ \\
Education level & 0.506 & $<0.001$ \\
$\begin{array}{l}\text { Impact of other stressful } \\
\text { events on mental health }\end{array}$ & 0.077 & 0.010 \\
$\begin{array}{l}\text { Lockdown duration } \\
\text { Impact of lockdown on }\end{array}$ & 0.424 & $<0.001$ \\
$\quad$ lifestyle & & $<0.001$ \\
SCOFF score & 0.550 & $<0.001$ \\
CAGE-AID & 0.250 & \\
\hline
\end{tabular}

during the last 3 months. The political and socio-economic crisis that the country has been going through since 2019 (Sly 2020; Hubbard 2020; Mounzer 2020) could explain this correlation, as many Lebanese had to adapt to massive changes to their quality of life. Indeed, unemployment, monthly income, professional status, having difficulties at work, having lost one's job, and having got financial difficulties were all found to be factors that significantly affected the measured variables. It is important to note that Lebanon responded well to the pandemic compared to other countries (Sly 2020), while the co-existing socio-economic crisis was not being so well managed (Mounzer 2020). This phenomenon might have exacerbated the impact of the socio-economic stressors compared to the pandemic and lockdown on the mental health of the Lebanese.

The practical implications of this study highlight the urgent need to develop and deliver more integrated policies and preventive measure aiming to support mental health during and after the current crisis. Such measures shall include efforts to increase the availability of mental health services on one hand, and secure incomes and jobs on the other hand. Policy makers must invest in mental health awareness-raising campaigns. Tips on coping strategies during such crises
Table 5 Risk factors associated with DASS scores identified by multiple Linear Regression Analysis

\begin{tabular}{llr}
\hline Variables & $\begin{array}{l}\text { Standardized } \\
\text { Coefficient }\end{array}$ & P-value \\
\hline Age & -0.104 & $\mathbf{< 0 . 0 0 1}$ \\
Monthly income & -0.052 & $\mathbf{0 . 0 3 1}$ \\
Education level & -0.102 & $<\mathbf{0 . 0 0 1}$ \\
Intensity of stressful life event on & 0.382 & $<\mathbf{0 . 0 0 1}$ \\
$\quad$ mental health & & \\
Impact of lockdown on lifestyle & 0.308 & $<\mathbf{0 . 0 0 1}$ \\
CAGE-AID score & 0.148 & $<\mathbf{0 . 0 0 1}$ \\
\hline
\end{tabular}

Model's adjusted $\mathrm{R}$ square $=0.403$

Model's p-value $<0.001$

The $\mathrm{Y}$ variable is the square root transform of DASS-21 score (which has a distribution not departing form normality assumptions)

$p$-values numbers marked in bold indicate numbers that are significant on the $95 \%$ confidence limit

shall be made available whether through sharing of mental health information or offering support hotlines. Governments should put in place actions in order to protect citizens on the employment and financial level; that would be done through helping in the transition to working from home, helping employers support the mental health of employees, especially those affected by the economic crisis, and offering training opportunities, job-search support, and counseling in order to support jobseekers back into employment. Finally access to mental health services either in face-to-face or tele-consultation shall be ensured and made available for the general population in order to provide support and treat to those who need it, and fill the gap that have been created by the pandemic.

Separation and Marital disputes were found to be significantly affecting the depression, anxiety, and stress variables, which is concordant with findings in the literature, as domestic violence was reported to have increased due to the lockdown measures in China (Whanqing 2020). Students were found to have higher depression, anxiety and stress
Fig. 1 Boxplots representing the correlation of DASS-21 total score with the impact of having been through a stressful life event on participant's mental health (right: $1=$ mild; $2=$ moderate; $3=$ severe; $4=$ very severe) and the intensity of lockdown's impact on participant's lifestyle (left: $0=$ absent; $1=$ mild; $2=$ moder ate; $3=$ severe)
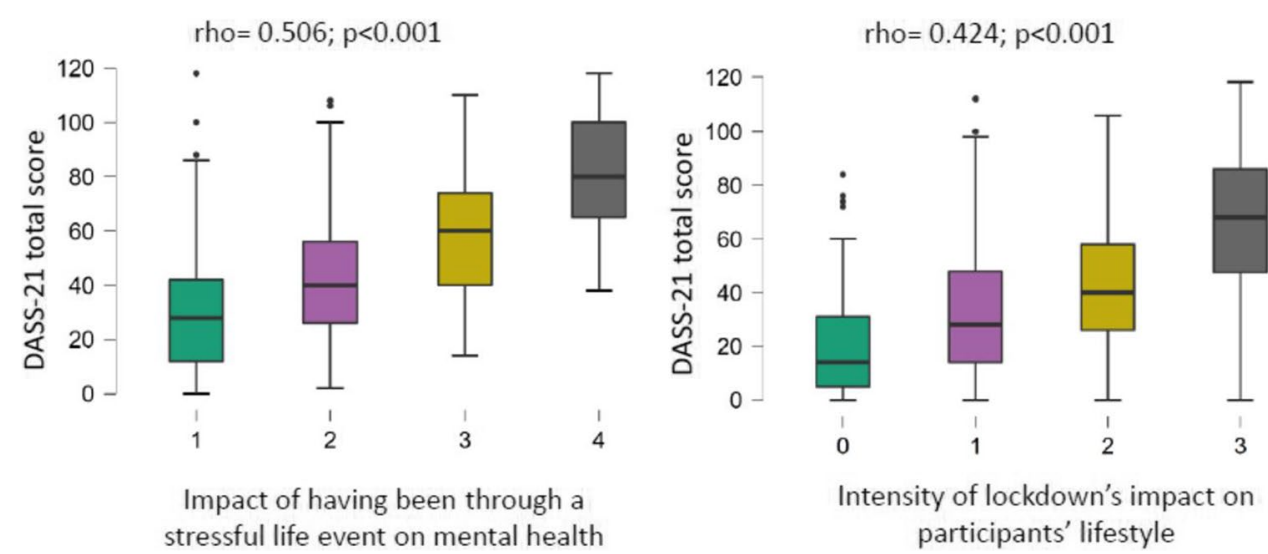
symptoms compared to employers/directors/managers which can be explained by a possible disruption to university life routine and the introduction of online courses and exams. In fact, psychological impact on students due to the COVID19 crisis had already been described in the literature (Wang et al., 2020a). Having a history of mental illness was found to be a significant risk factor affecting the measured symptoms which underlines the importance of providing mentalhealth services in times of pandemic and lockdown, as mentally-ill people could be at a higher risk of relapse. Future studies are essential to evaluate the impact of lockdown and pandemics on patients with mental illnesses.

Factors related to socio-demographic parameters affecting the DASS-21 score in the regression model show that participants with a higher age, a higher income, a better professional status and a better education level had less severe scores. This is in concordance with previous data demonstrating that having a younger age and a lower socioeconomic status are considered to be important contextual risk factors for depression and anxiety in the general population in addition to other risk factors such as female gender, domestic violence, working in healthcare system, etc. (Razai, et al. 2020; Pappa, et al. 2020). Although we had more respondents with a higher socio-economic level, the fact that our sample found that socio-economic stressors were more impactful than the impact of the lockdown and the pandemic per se, suggests that subjects of low socio-economic level should intuitively, have at least the same level of impact due to the economic crisis, or even more.

Our study had some limitations. First, it is a cross-sectional study, and conclusive causal relationships cannot be inferred. Second, the use of subjective self-reported questionnaires might have lacked the validity of face-to-face interviews. The use of a web-based questionnaire might also be a selection/response bias. As the questionnaires were distributed via social media outlets, this study does not include potential responders who do not have internet connection or do not use social media. Other non-responders were too stressed to participate or did not feel concerned by this study. Third, the internal consistency of the SCOFF questionnaire was not satisfactory and, accordingly, it has not been considered in the multivariate analysis. Caution should thus be practiced in generalizing the results to the Lebanese population.

\section{Conclusion}

In summary, our findings contribute to the understanding of the psychological impact of the COVID-19 outbreak in a sample of the general population in Lebanon and outline a significant impact of lockdown, as well as other socioeconomic stressors on depression, anxiety and stress scores.
However, in the setting of the COVID-19 pandemic in the Lebanese population, we have demonstrated that socio-economic stress might be more impacting the Lebanese citizens' psychological wellbeing than any stress related to the pandemic and its related repercussion on lifestyle. Future studies are essential to evaluate the prospective long-term psychological outcomes in this population. Our findings nevertheless suggest the importance of socio-economic interventions and a multidisciplinary approach in policymaking that also targets additional stressors accompanying a pandemic as both may have a major impact on mental health and wellbeing.

Acknowledgements We express our gratitude to all those who participated in this study.

Funding This research did not receive any specific grant from funding agencies in the public, commercial, or not-for-profit sectors.

Data availability The datasets generated during and/or analysed during the current study are available from the corresponding author on reasonable request.

\section{Declarations}

Ethics Approval The questionnaire and methodology for this study was approved by the Human Research Ethics committee of Hotel Dieu de France hospital (Ethics approval number: CEHDF1632).

Conflict of interest The authors declare that there are no conflicts of interest.

\section{References}

Brooks, S. K., Webster, R. K., Smith, L. E., et al. (2020). The psychological impact of quarantine and how to reduce it: Rapid review of the evidence. The Lancet, 395, 912-920. https://doi.org/10.1016/ S0140-6736(20)30460-8

Brown, R. L., \& Rounds, L. A. (1995). Conjoint screening questionnaires for alcohol and other drug abuse: Criterion validity in a primary care practice. Wisconsin Medical Journal, 94, 135-140.

Bo, H.-X., Li, W., Yang, Y., et al. (2020). Posttraumatic stress symptoms and attitude toward crisis mental health services among clinically stable patients with COVID-19 in China. Psychological Medicine, 1-2,. https://doi.org/10.1017/S0033291720000999

Cao, W., Fang, Z., Hou, G., et al. (2020). The psychological impact of the COVID-19 epidemic on college students in China. Psychiatry Research, 287, 112934. https://doi.org/10.1016/j.psychres.2020. 112934

Dong, L., \& Bouey, J. (2020). Public Mental Health Crisis during COVID-19 Pandemic China. Emerging Infectious Diseases, 26,. https://doi.org/10.3201/eid2607.200407

Hagerty, S. L., \& Williams, L. M. (2020). The impact of COVID-19 on mental health: The interactive roles of brain biotypes and human connection. Brain, Behavior and Immunity, 5, 100078. https://doi. org/10.1016/j.bbih.2020.100078

Hubbard (2020) Lebanon's Economic Crisis Explodes, Threatening Decades of Prosperity. New York Times 
Jiang, L., Yan, Y., Jin, Z.-S., et al. (2020). The Depression Anxiety Stress Scale-21 in Chinese Hospital Workers: Reliability, Latent Structure, and Measurement Invariance Across Genders. Frontiers in Psychology, 11,. https://doi.org/10.3389/fpsyg.2020.00247

Jin, Y., Yang, H., Ji, W., et al. (2020). Virology, Epidemiology, Pathogenesis, and Control of COVID-19. Viruses, 12, 372. https://doi. org/10.3390/v12040372

Karam, E. G., Mneimneh, Z. N., Dimassi, H., et al. (2008). Lifetime Prevalence of Mental Disorders in Lebanon: First Onset, Treatment, and Exposure to War. PLoS Medicine, 5,. https://doi.org/ 10.1371/journal.pmed.0050061

Ko, C.-H., Yen, C.-F., Yen, J.-Y., \& Yang, M.-J. (2006). Psychosocial impact among the public of the severe acute respiratory syndrome epidemic in Taiwan. Psychiatry and Clinical Neurosciences, 60, 397-403. https://doi.org/10.1111/j.1440-1819.2006.01522.x

Lai, J., Ma, S., Wang, Y., et al. (2020). Factors Associated With Mental Health Outcomes Among Health Care Workers Exposed to Coronavirus Disease 2019. JAMA Network Open, 3, e203976. https:// doi.org/10.1001/jamanetworkopen.2020.3976

Lau, J. T., Griffiths, S., Choi, K. C., \& Tsui, H. Y. (2010). Avoidance behaviors and negative psychological responses in the general population in the initial stage of the H1N1 pandemic in Hong Kong. BMC Infectious Diseases, 10, 139. https://doi.org/10.1186/ 1471-2334-10-139

Le, M. T. H., Tran, T. D., Holton, S., et al. (2017). Reliability, convergent validity and factor structure of the DASS-21 in a sample of Vietnamese adolescents. PLoS ONE, 12, https://doi.org/10.1371/ journal.pone. 0180557

Leigh-Hunt, N., Bagguley, D., Bash, K., et al. (2017). An overview of systematic reviews on the public health consequences of social isolation and loneliness. Public Health, 152, 157-171. https://doi. org/10.1016/j.puhe.2017.07.035

Leung, G. M. (2003). The impact of community psychological responses on outbreak control for severe acute respiratory syndrome in Hong Kong. Journal of Epidemiology and Community Health, 57, 857-863. https://doi.org/10.1136/jech.57.11.857

Liu, S., Yang, L., Zhang, C., et al. (2020). Online mental health services in China during the COVID-19 outbreak. Lancet Psychiatry, 7, e17-e18. https://doi.org/10.1016/S2215-0366(20)30077-8

Lovibond SH, Lovibond PF, Australia PF of (1995) Manual for the depression anxiety stress scales. Sydney, N.S.W. : Psychology Foundation of Australia

Mengin, A., Allé, M. C., Rolling, J., et al. (2020). Psychopathological consequences of confinement. L'encephale. https://doi.org/10. 1016/j.encep.2020.04.007

Mollica, R. F. (1993). The Effect of Trauma and Confinement on Functional Health and Mental Health Status of Cambodians Living in Thailand-Cambodia Border Camps. JAMA, 270, 581. https://doi. org/10.1001/jama.1993.03510050047025

Morgan, J. F., Reid, F., \& Lacey, J. H. (1999). The SCOFF questionnaire: Assessment of a new screening tool for eating disorders. BMJ, 319, 1467-1468. https://doi.org/10.1136/bmj.319.7223. 1467

Mounzer L (2020) Opinion I In Lebanon, a Pandemic of Hunger. New York Times
Pappa, S., Ntella, V., Giannakas, T., et al. (2020). Prevalence of depression, anxiety, and insomnia among healthcare workers during the COVID-19 pandemic: A systematic review and meta-analysis. Brain, Behavior and Immunity, 88, 901-907. https://doi.org/10. 1016/j.bbi.2020.05.026

Razai, M. S., Oakeshott, P., Kankam, H., et al. (2020). Mitigating the psychological effects of social isolation during the covid-19 pandemic. BMJ, 369,. https://doi.org/10.1136/bmj.m1904

Sly (2020) Lebanon is in a big mess. But on coronavirus, it's doing something right. Washimgton Post

Sønderskov, K. M., Dinesen, P. T., Santini, Z. I., \& Østergaard, S. D. (2020). Increased psychological well-being after the apex of the COVID-19 pandemic. Acta Neuropsychiatrica, 32, 277279. https://doi.org/10.1017/neu.2020.26

Sprang, G., \& Silman, M. (2013). Posttraumatic Stress Disorder in Parents and Youth After Health-Related Disasters. Disaster Medicine and Public Health Preparedness, 7, 105-110. https://doi.org/10. 1017/dmp.2013.22

Taylor, M. R., Agho, K. E., Stevens, G. J., \& Raphael, B. (2008). Factors influencing psychological distress during a disease epidemic: Data from Australia's first outbreak of equine influenza. $B M C$ Public Health, 8, 347. https://doi.org/10.1186/1471-2458-8-347

Torales, J., O’Higgins, M., Castaldelli-Maia, J. M., \& Ventriglio, A. (2020). The outbreak of COVID-19 coronavirus and its impact on global mental health. International Journal of Social Psychiatry, 66, 317-320. https://doi.org/10.1177/0020764020915212

Wang, C., Pan, R., Wan, X., et al. (2020a). Immediate psychological responses and associated factors during the initial stage of the 2019 coronavirus disease (COVID-19) epidemic among the general population in China. International Journal of Environmental Research and Public Health, 17(5). https://doi.org/10.3390/ijerp h17051729

Wang H, Xia Q, Xiong Z, et al. (2020b). The psychological distress and coping styles in the early stages of the 2019 coronavirus disease (COVID-19) epidemic in the general mainland Chinese population: a web-based survey. medRxiv. https://doi.org/10.1101/2020. 03.27.20045807

Vindegaard, N., \& Benros, M. E. (2020). COVID-19 pandemic and mental health consequences: Systematic review of the current evidence. Brain, Behavior and Immunity, 89, 531-542. https:// doi.org/10.1016/j.bbi.2020.05.048

Whanqing (2020) Domestic Violence Cases Surge During COVID-19 Epidemic. In: Sixth Tone. https://www.sixthtone.com/news/domes tic-violence-cases-surge-during-covid-19-epidemic. Accessed 6 Jun 2020

Zandifar, A., \& Badrfam, R. (2020). Iranian mental health during the COVID-19 epidemic. Asian Journal of Psychiatry, 51, 101990. https://doi.org/10.1016/j.ajp.2020.101990

Publisher's note Springer Nature remains neutral with regard to jurisdictional claims in published maps and institutional affiliations. 